\title{
Ferroelectric "gourd" goes into vdW atomic cage
}

Shuai Dong

School of Physics, Southeast University, Nanjing 211189, China

E-mail: sdong@seu.edu.cn

$\mathrm{F}$ erroelectric materials have been discovered for one century and widely served in many devices, especially in micro-electro-mechanical systems. With increasing demands for device miniaturization, the ferroelectricity persisting to nanoscale and below becomes more and more crucial, but remains a challenging scientific question. Although cutting-edge molecular bean epitaxy and pulsed laser deposition techniques can grow thin films down to atomic level, the surface charges from dangling bonds, uncompensated depolarization field, as well as epitaxial substrates, can easily kill their ferroelectricity and lead to the notorious "dead layer".

The van der Walls (vdW) materials bring a grand opportunity to the ferroelectric community. Comparing with ferroelectric oxides with compact three-dimensional (3D) framework, these layered materials are natural rich ore for atomic sheets. Monolayer or few-layers can be easily exfoliated from bulks thanks to the weak coupling between layers. Their saturated surfaces are more chemically passivated, which can avoid serious surface reconstruction after exfoliation and mostly retain their original properties. Even though, the depolarization effect remains a challenge question if their polarizations point perpendicular to the sheets.

In 2016, Wang, Liu, and their collaborators, for the first time, observed the out-of-plane ferroelectric polarization in $\mathrm{CuInP}_{2} \mathrm{~S}_{6}$ few layers [1]. A little earlier, Ji and his collaborators observed the in-plane ferroelectric domain in SnTe monolayer [2]. These pioneer works opened an era of two-dimensional vdW ferroelectrics. Since then, more and more vdW ferroelectrics have been explored [3]. New interesting properties and novel mechanisms of polarity beyond conventional 3D counterparts have also been revealed [4].

$\mathrm{CuInP}_{2} \mathrm{~S}_{6}$ is a unique system, whose polarization is generated by copper-indium pairs (see Fig. 1) caged in loose phosphorus-sulfur framework. The charge asymmetry of copper-indium pairs is switchable within the cage. Surprisingly, the copper can even move outside the cage a little, leading to rare quadruple polarization states [5,6]. Moreover, the combination between "hard" phosphorus-sulfur framework and "soft" vdW

\footnotetext{
$*_{\text {Received September 8, 2020. This article also can be found at }}$ http://journal.hep.com.cn/fop/EN/10.1007/s11467-020-0998-9
}

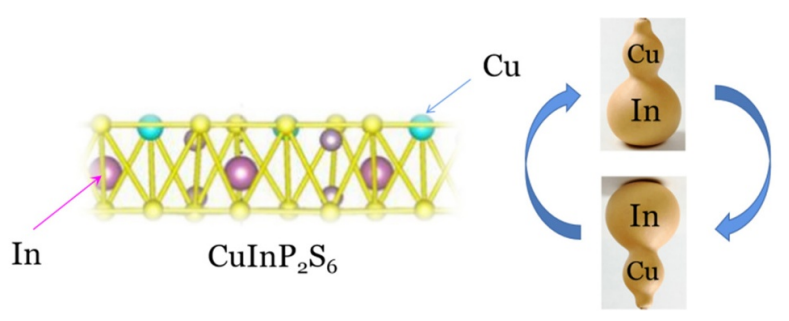

Fig. 1 Schematic of $\mathrm{CuInP}_{2} \mathrm{~S}_{6}$ and its ferroelectric switching.

layer lead to exotic negative piezoelectricity, which is the second experimentally confirmed one (and the first in inorganic materials) [5]. Also, thanks to the high mobility of small copper ions and loose framework, the ionic conduction is prominent in this system [7], an uncommon property for ferroelectric materials and important for ionic battery applications.

Recently, You, Wang, and their collaborators systematically reviewed the interesting physics of $\mathrm{CuInP}_{2} \mathrm{~S}_{6}$ and its latest progress [8].

\section{References}

1. F. Liu, L. You, K. L. Seyler, X. Li, P. Yu, J. Lin, X. Wang, J. Zhou, H. Wang, H. He, S. T. Pantelides, W. Zhou, P. Sharma, X. Xu, P. M. Ajayan, J. L. Wang, and Z. Liu, Room-temperature ferroelectricity in $\mathrm{CuInP}_{2} \mathrm{~S}_{6}$ ultrathin flakes, Nat. Commun. 7, 12357 (2016)

2. K. Chang, J. Liu, H. Lin, N. Wang, K. Zhao, A. Zhang, F. Jin, Y. Zhong, X. Hu, W. Duan, Q. Zhang, L. Fu, Q. K. Xue, X. Chen, and S. H. Ji, Discovery of robust in-plane ferroelectricity in atomic-thick SnTe, Science 353, 274 (2016)

3. M. Wu and P. Jena, The rise of two-dimensional van der Waals ferroelectrics, WIREs Comput. Mol. Sci. 8, e1365 (2018)

4. L.-F. Lin, Y. Zhang, A. Moreo, E. Dagotto, and S. Dong, Frustrated dipole order induces noncollinear proper ferrielectricity in two dimensions, Phys. Rev. Lett. 123, 067601 (2019)

5. L. You, Y. Zhang, S. Zhou, A. Chaturvedi, S. A. Morris, F. Liu, L. Chang, D. Ichinose, H. Funakubo, W. Hu, T. Wu, Z. Liu, S. Dong, and J. L. Wang, Origin of giant negative piezoelectricity in a layered van der Waals ferroelectric, Sci. Adv. 5(4), eaav3780 (2019)

6. J. A. Brehm, S. M. Neumayer, L. Tao, A. O'Hara, M. Chyasnavichus, M. A. Susner, M. A. McGuire, S. V. Kalinin, S. Jesse, P. Ganesh, S. T. Pantelides, P. Maksymovych, and N. Balke, Tunable quadruple-well ferroelectric van der Waals crystals, Nat. Mater. 19, 43 (2020)

7. S. Zhou, L. You, A. Chaturvedi, S. A. Morris, J. S. Herrin, N. Zhang, A. Abdelsamie, Y. Hu, J. Chen, Y. Zhou, S. Dong, and J. L. Wang, Anomalous polarization switching and permanent retention in a ferroelectric ionic conductor, Mater. Horiz. 7, 263 (2020)

8. S. Zhou, L. You, H. L. Zhou, Y. Pu, Z. G. Gui, and J. L. Wang, Van der Waals layered ferroelectric $\mathrm{CuInP}_{2} \mathrm{~S}_{6}$ : Physical properties and device applications, Front. Phys. 16(1), 13301 (2021) 